\title{
Study of the electronic phase transition with low dimensionality in $\mathrm{SrVO}_{3}$ thin films
}

Arnaud Fouchet ${ }^{1}$, Mickaël Allain ${ }^{1}$, Bruno Bérini ${ }^{1}$, Elena Popova ${ }^{1}$, Pierre-Eymeric Janolin ${ }^{2}$, Nicolas Guiblin $^{2}$, Ekaterina Chikoidze ${ }^{1}$, Joseph Scola ${ }^{1}$, David Hrabovsky ${ }^{1}$, Yves Dumont ${ }^{1}$, Niels Keller ${ }^{1}$

${ }^{1}$ Université Versailles St Quentin, CNRS, Université Paris-Saclay, Groupe d'Étude de la Matière Condensée (GEMaC), 78035 Versailles, France. *Email: fouchet@physique.uvsq.fr

${ }^{2}$ Université Paris Saclay, Laboratoire Structure Proprietes \& Modelisations Solides, CNRS, Centrale Supelec, F-92295 Chatenay Malabry, France

(Received $\mathrm{xx}$ xxxx xxx; published $\mathrm{xxxx} \mathrm{xxxxx}$ )

\begin{abstract}
Transport and structural properties of ultrathin films of $\mathrm{SrVO}_{3}(\mathrm{SVO})$ on $\mathrm{SrTiO}_{3}(001)$ substrates have been investigated and correlations between Metal-Insulator Transition (MIT) and strain relaxation have been studied. Below a critical thickness, when the film is subjected to tensile strain, the resistivity of the films is increasing with decreasing film thickness. Transport properties evolve from metallic to strongly localized state in several monolayer thick films, showing the bandwidth $W$ control of the Mott-Hubbard transition with the film thickness. Furthermore, a dimensional crossover from 3 Dimensions to 2 Dimensions has been studied by transport measurements. Using Quantum Corrections to the Conductivity (QCC), it is demonstrated that MIT is due to renormalized electron-electron interaction in this material. Finally, for films with the thickness below $6 \mathrm{~nm}$, the confinement provides new effect in magnetotransport with apparition of weak antilocalization in ultrathin films.
\end{abstract}

\section{Introduction}

These last decades, transition metal perovskites have shown a wealth of novel and functional properties such as high $\mathrm{T}_{\mathrm{C}}$ superconductivity, colossal magnetoresistance and full spin polarization in conductors. In these strongly correlated electron materials, the physical properties are driven through the strong interplay between charge, spin, orbital and lattice degree of freedom. Among them, metallic $\mathrm{SrVO}_{3}$ is a normal Fermi liquid and can be driven into a Mott insulator state by reducing its dimensionality and through strain engineering. 
First synthesized in 1957 [1], Strontium Vanadate $\left(\mathrm{SrVO}_{3-\delta}\right)$ presents a cubic perovskite structure with a cell parameter $a=3.843 \AA$ for $\delta=0$ and $a=3.856 \AA$ corresponding to $\delta=0.1$ with a space group $P m$ $3 m[2,3]$. Moreover, physical properties of $\mathrm{SrVO}_{3-\delta}$ have been widely studied [4-6]. This material presents a robust metallic behavior, independent of the oxygen stoichiometry content and an enhanced Pauli paramagnetic character, both for bulk and thin films.

Most recently, with first principles methods, a Metal-Insulator Transition (MIT) in SVO heterostructures has been predicted for 4-5 unit-cell thicknesses. Furthermore, the strontium vanadate in insulating state is expected to be a Mott insulator and ferromagnetic below this critical thickness [7].

According to the Mott-Hubbard theory [8], MIT is controlled by the ratio of the on-site Coulomb repulsion $U$ and the electronic bandwidth $W$. In perovskites, the bandwidth is influenced by the overlapping of the $3 d$ orbitals of the TM (TM: Transition Metal) with the oxygen $2 p(O)$ orbitals, which is determined by the bond angle of $\mathrm{O}$ ions with TM ion and the TM-O bond length. Bandwidthcontrolled MIT (BC-MIT) of SVO has already been realized by chemical substitutions in $\mathrm{Ca}_{x} \mathrm{Sr}_{1-\mathrm{x}} \mathrm{VO}_{3}: \mathrm{Ca}$ substitution on the A site (Ca cation being smaller than $\mathrm{Sr}$ with the same valence) controls the bandwidth $\mathrm{W}$ and the strength of the electron correlation $U / W$ without changing the band filling $[9,10]$. Indeed, the O-V-O angle is $180^{\circ}$ in $\mathrm{SVO}$ and $160^{\circ}$ for $\mathrm{CaVO}_{3}$ and is very close to the angle of MIT. Another approach to control $3 \mathrm{~d}-\mathrm{W}$ is dimensional cross-over in ultrathin films that has been shown experimentally by photoemission spectroscopy $[5,11]$. In the work of Yoshimatsu et al. [5], the studies of the V3d states at Fermi energy $\left(\mathrm{E}_{\mathrm{F}}\right)$ have evidenced the evolution of the electronic structure with the formation of a pseudo-gap at $E_{F}$ for thickness of 3-6 monolayers (ML) and the opening of a Mott-Hubbard gap at 2-3ML. The effective bandwidth $W$ has been driven by the reduction of the effective coordination number at the interface and the surface. 
In the present work we have studied the correlation between mechanical strain and electronic properties of the SVO heterostructures and the emergence of the MIT at dimensional cross-over by transport measurements.

Since transport properties in SVO have been mostly studied for metallic films, measurements of these properties for ultrathin films with thicknesses in the MIT range (from 2 to 15 unit-cells) represent an important issue to understand the origin of the MIT in this material. Similar studies have been realized for oxide perovskites such as $\mathrm{LaNiO}_{3}[12,13]$ or $\mathrm{SrRuO}_{3}[12,14]$ and give clue to understand the origin of the strong localization behavior of carriers for these materials.

The transport measurements carried out on our SVO thin films have shown appearance of MIT for ultrathin films. Theoretical models will be used in order to understand its origin which cannot be described using only phenomenological Mathiessen [15] equation of the resistivity $\rho=\rho_{0}+A * T^{n}$, with $\mathrm{n}$ depending on the collision mechanism. The description of the evolution of conductivity which describes the quantum corrections to the Drude conductivity in disordered metals is designated as Quantum Corrections of the conductivity (QCC) [16]. Two main contributions are used to explain these QCC: i) the first one is the Renormalized Electron-Electron Interaction (REEI) and corresponds to the density of state correction at the Fermi energy level, ii) the second is the Weak Localization (WL) and corresponds to interference effect due to defects or impurities scattering. Lee and Ramakrishnan [16] have established that for a two-dimensional system (2D) both REEI and WL effects give a logarithmic temperature dependence of the conductivity:

$$
\sigma(T)=\sigma_{0}+p * \frac{e^{2}}{\pi * h} \ln \left(T / T_{0}^{\prime}\right) \text { for WL, } \quad \text { and } \quad \sigma(T)=\sigma_{0}+B * \frac{e^{2}}{2 \pi * h} \ln \left(T / T_{e}\right) \text { for REEI }
$$

Where e is charge of the electron, $\mathrm{h}$ is the Planck's constant, $T_{0}^{\prime}$ is related to the transport mean free path $\sigma_{0}$ is the Drude conductivity and $\mathrm{p}$ discriminates the main inelastic relaxation mechanism between electron-electron scattering $(p=1)$ or electron-phonon scattering $(p=3), B$ term 
accounts for the scattering parameter of the Coulomb interaction and $T_{e}$ is the minimum experimental temperature [17].

Whereas $\ln (3 D)$ :

$\rho(T)=\frac{1}{\sigma_{0}+a_{1} * T^{\frac{q}{2}}+a_{2} * T^{\frac{1}{2}}}+A * T^{2}$

different terms for WL and REEI account for the quantum correction to the resistivity and could be discriminated: WL effects correspond to the $\mathrm{a}_{1}{ }^{*} \mathrm{~T}^{\mathrm{q} / 2}$ term (with $\mathrm{q}=2$ for electron-electron interactions, $q=3$ for electron-phonon scattering), while REEl is related to the $a_{2} * T^{1 / 2}$ term. Moreover, an additional term $A * T^{2}$ accounts for electron correlation effects in Fermi liquid description on the resistivity.

\section{Experimental}

$\mathrm{SrVO}_{3}$ thin films were grown on $\mathrm{TiO}_{2}$-terminated (100) $\mathrm{SrTiO}_{3}$ (STO) substrates by Pulsed Laser Deposition (PLD) in $5^{*} 10^{-6}$ Torr oxygen pressure at $710^{\circ} \mathrm{C}$. A $248 \mathrm{~nm}$ wavelength $\mathrm{KrF}$ excimer laser was employed with a repetition rate of $1 \mathrm{~Hz}$ and a fluency of $250 \mathrm{~mJ}$. During the growth, surface structure was characterized by Reflection High-Energy Electron Diffraction (RHEED) and the film thickness was measured with ex-situ X-Ray Reflectivity (XRR) and Laue oscillations. Structural relaxation of thin films has been observed through the measurements of both in-plane and out-ofplane lattice parameter. The latter was measured by X-Ray Diffraction (XRD) in $\theta-2 \theta$ configuration. Topography and roughness of the films have been investigated by atomic force microscopy (AFM) (Bruker Dimension 3100) in tapping mode using commercial tips with $300 \mathrm{kHz}$ resonant frequency and $40 \mathrm{~N} / \mathrm{m}$ spring constant. Transport properties were measured using four in-line aluminium pad contact geometry and van der Pauw method in a Physical Properties Measurement System (PPMS) in the $2 \mathrm{~K}-300 \mathrm{~K}$ temperature range. 


\section{Results and discussions}

Structural characterizations:

Fig. 1(a) shows the evolution of the out-of-plane lattice parameter of thin films versus thickness. Film thicknesses have been calculated from finite size Laue oscillations observed around (00l) symmetric reflections of films and substrates as shown in the inset to fig. 1(a) for planes (002) of $9 \mathrm{~nm}$ and 53.5 $\mathrm{nm}$ thin films. These thickness fringes indicate the sharp interface and a very smooth and coherent film and confirm the structural quality of the films. Fits using a dynamical model have been carried out since it has been reported [18] that more accurate results are obtained in case of ultrathin films due to the signal intensity coming from substrate/film interference. These fits give access to both the thickness and out-of-plane lattice parameter which was extracted from the average of the fit around the (00l) reflections peak in order to minimize errors. The flatness of the film was confirmed by AFM topographic image as shown on the figure SI-1 for the SVO film of $3 \mathrm{~nm}$. Terraces can be observed corresponding to the same morphology than the STO substrate. The rough mean square of the film is $0.2 \mathrm{~nm}$.

For SVO thicker than $50 \mathrm{~nm}$, the films are fully relaxed with an out-of-plane lattice parameter slightly bigger than the bulk (see $\mathrm{SrVO}_{3}$ lattice parameter line on fig. 1a). As seen previously [3], this phenomenon can be explained by a small non-stoichiometry in oxygen (in between $\mathrm{SrVO}_{3}$ and $\mathrm{SrVO}_{2.9}$ [2]), resulting from the low oxygen pressure during the growth [19]. For thicknesses below $50 \mathrm{~nm}$, the out-of-plane lattice parameter begins to decrease and shows the progressive influence of the tensile strain $(-1.6 \%)$ imposed by the STO substrate. Due to small intensity of the X-Ray diffraction peaks for films thinner than $9 \mathrm{~nm}$, RHEED has been used in order to extract the in-plane lattice parameter along [110] and [100] directions. The inset to fig. 1(b) shows a RHEED pattern recorded during the growth. The $d_{-}\{110\}$ interplanar distance versus film thickness was extracted from these RHEED patterns during the growth and is presented in the fig. 1(b), where variations with film thickness can be observed. At the first steps of the growth (between 0 and $6 \mathrm{~nm}$ ), an overshoot 
of the d_ $\{110\}$ interplanar distance is measured. This effect has already been observed in similar growth and is explained by the surface atom displacements due to the strong lattice relaxation via the formation of the dislocations [20,21]. Above the thickness of $6 \mathrm{~nm}$, the in-plane lattice parameter seems to be more or less constant in the measurement uncertainty whereas slower relaxation of the out-of-plane lattice parameter is evidenced up to $50 \mathrm{~nm}$ (see fig1(a)).

Physical properties:

Fig. 2(a) presents the evolution of resistivity versus temperature for different sample thicknesses. Films thicker than $50 \mathrm{~nm}$ are metallic and their resistivities are comparable. Below this thickness, down to $7 \mathrm{~nm}$, resistivity continuously increases as the thickness is reduced although the films are metallic in the whole temperature range. Below $7 \mathrm{~nm}$ and down to about $3 \mathrm{~nm}$, an upturn appears on the curves at $10 \mathrm{~K}, 38 \mathrm{~K}$ and $85 \mathrm{~K}$ for $7 \mathrm{~nm}, 6 \mathrm{~nm}$ and $3 \mathrm{~nm}$ films, respectively. For films thinner than 1.5-2 $\mathrm{nm}$ an insulating behavior is found at all temperatures.

For metallic films which correspond to thicknesses between $350 \mathrm{~nm}$ and $13 \mathrm{~nm}$, resistivity shows a quadratic temperature dependence $\rho=\rho_{0}+A * T^{2}$, (where $\mathrm{A}$ is related to electron-electron scattering and $\rho_{0}$ is the background contribution due to static disorder [9]). This dependence is characteristic of Fermi liquid behavior in correlated electrons and is a common feature observed for several metallic oxide perovskites [6,9,22-25]. As shown in fig. 2(b) for the $115 \mathrm{~nm}$ thick film, this model describes perfectly the data in the whole temperature range and parameter values are $\rho_{0}=2.7 * 10^{-5} \Omega . \mathrm{cm}$ and $A=2.2 * 10^{-10} \Omega . \mathrm{cm} . K^{-2}$. Table 1 summarizes the A and $\rho_{0}$ values corresponding to SVO metallic films for the various thicknesses. Our values for thin films in 13-350 $\mathrm{nm}$ thickness range are consistent with these reported for bulk SVO materials for which $\mathrm{A}$ is ranging from $4.0 * 10^{-10} \Omega . \mathrm{cm} \cdot K^{-2}[9,22]$ to $6.0 * 10^{-8} \Omega . \mathrm{cm} \cdot K^{-2}$ [6]. In the Fermi liquid model the residual resistivity $\rho_{0}$ leads to values between $6.0 * 10^{-6} \Omega . \mathrm{cm}[9,22,26]$ and $3.0 * 10^{-2} \Omega . \mathrm{cm}[6]$, thus our values presented in table 1 are showing that the quality of our SVO films are comparable to 
SVO single crystal and thin films. Nevertheless, the ratio $\rho_{300 K} / \rho_{2 K}<2$ [27] is also indicating a slight non-stoichiometry in cation or oxygen and could explain the appearance of half-order streaks on the RHEED patterns (fig. 1b) [28].

When the film thickness is reduced to $7 \mathrm{~nm}, 6 \mathrm{~nm}$ and $3 \mathrm{~nm}$, a low temperature upturn appears at different temperatures. For these films, the metallic part follows as well a $\mathrm{T}^{2}$ law and the parameters $\rho_{0}$ and $A$ tend to increase with the decrease of the thickness (see table 1 ).

Fig. 2(d) presents the low-temperature behavior of the resistivity for the $7 \mathrm{~nm}$ SVO film and the corresponding 3D fit calculated from eq. (2). For this thickness, an upturn appears below $10 \mathrm{~K}$, showing the QCC. This 3D model tends to reproduce the increase of the resistivity at low temperature. Interestingly, the $a_{1} * T^{\frac{q}{2}}$ term, which represent the weak localization (WL), tends to zero whereas the $a_{2}{ }^{*} T^{1 / 2}$ term, which is the REEI contribution, contributes mainly to the resistivity upturn. To go further in the determination of the transport mechanism dimension and to discriminate with a possible 2D system, we show in the inset of fig. 2(d) the sheet conductance evolution as a function of $\ln (\mathrm{T})$ in the temperature range where an upturn is observed. It appears clearly that for the two models corresponding to WL mechanisms calculated from the eq. (1) with electron-electron scattering $(p=1)$ or electron-phonon scattering $(p=3)$, a WL 2D regime is not able to describe the experimental data. However, the fit, obtained using the equation of 2D REEI mechanism, describes successfully the data as corresponding to the 3D model. Finally, for this thickness, although it is difficult to conclude on the dimensionality of the system, the main contribution to the resistivity upturn is the REEI mechanism.

Fig. 3(a) and its inset present the low temperature behavior of the resistivity versus temperature and the evolution of the sheet conductance as a function of $\ln (T)$ for a $3 \mathrm{~nm}$ SVO film respectively. The 3D 
model calculated from the eq. (2) is plotted in fig. 3(a) and a very good agreement with the experimental data is observed. As for the $7 \mathrm{~nm}$ SVO thin film, the WL contribution tends to zero, therefore mainly the REEI contributes to the changes in transport properties. The inset of fig. 3(a) shows the 2D models for WL and REEI. In this case, REEI and WL (with $p=1$ ) models agree with the data. However, for both of them, the fit deviates for temperature above $20 \mathrm{~K}(\ln T=3)$. As the temperature increases, an additional electron-phonon scattering effect explain the phenomena $(p=3)$ and a coexistence with the REEI 2D describes the results of the transport measurements.

For a 1.5-2 nm SVO thin film, (shown in fig. 2(a)), as the previous model failed, electron localization effect have been taken into account with a Mott Variable Range Hopping (VRH) model for the conductivity [29]. In this model (see eq. 3), electrons hop between localized states and $\alpha=\frac{1}{d+1}$ is describing the system dimension $(d) . \mathrm{T}_{0}$ is a physical parameter proportional to the density of states at Fermi energy $E_{\mathrm{F}}$ and the localization length of the charge carriers.

$\sigma=\sigma_{0} * \exp \left[\left(-T_{0} / T\right)^{\alpha}\right]$

Fig. 3(b) presents the logarithm of sheet conductance as a function of $1 / \mathrm{T}^{1 / 3}$ and the calculated model for a 2D system with $\alpha=1 / 3$. Since a 3D fit (with $\alpha=1 / 4$ ) has shown less accuracy, we present here $2 \mathrm{D}$ system only. In the $2 \mathrm{~K}-40 \mathrm{~K}$ range, the agreement with the linear fit is excellent and results in parameter values $\sigma_{0}=0.32 \pm 0.01 \mathrm{mS}$. sq and $T_{0}=13.2 \mathrm{~K}$. The values for $\sigma_{0}$ are in the same order as VRH results for a $2 \mathrm{~nm} \mathrm{LaNiO}_{3}$ film reported by Scherwitzl et al. [13] with $\sigma_{0}=0.13 \mathrm{mS}$. sq, the other parameter value being $T_{0}=4.0 \mathrm{~K}$.

To go further in the interpretation of transport measurements, studies of the conduction mechanism have also been investigated by magnetoresistance (MR) measurements. The MR has been measured in perpendicular field for all the films and a positive magnetoresistance has been obtained. For example, MR of the $3 \mathrm{~nm}$ thick film is shown in fig. $4(\mathrm{a})$ for different temperature and its magnitude is 
decreasing with increasing temperature. Moreover, $\mathrm{MR}$ for the $3 \mathrm{~nm}$ have been fitted using $\mathrm{H}^{2}$ dependence and perfect superposition of the fit and the experimental data is obtained for high fields $H>3 T$. These measurements are consistent with REEl and the dependency of $H^{2}$ is attributed to the Lorentz force. Furthermore, Hall measurements are shown on the figure $2 c$ for the films with a thickness of $115 \mathrm{~nm}$ and $3 \mathrm{~nm}$. In both cases, the carrier's type is electrons for all the temperature. For the $115 \mathrm{~nm}$ thick film, the carrier concentration is $3.10^{22} \mathrm{~cm}^{-3}$ whereas for the $3 \mathrm{~nm}$ thick film the carrier concentration is half this value. Furthermore for the thick film, the carrier concentration stays constant whereas for the thin film, the carrier concentration tends to decrease with the decrease of the temperature. In both case the electron mobility slightly increases as the temperature decreases with a small decrease below $25 \mathrm{~K}$ for the $3 \mathrm{~nm}$ thick film. Interestingly, it is possible to differentiate the localization and interactions effects from the Hall coefficient $R_{H}$. Whereas the Hall coefficient $\left(R_{H}\right)$ $\left(n=1 /\left(R_{H}^{*} e\right)\right.$ ) (with $n$ the carrier density and e the charge of the electron), $R_{H}$ is constant for the localization effect and was found to increase logarithmically at a rate equal to twice that of the resistivity at low temperature [16]. We observe a decrease of the carrier density with the decrease of the temperature which invalids the dependency of $R_{H}$ for localization effect. Thus, this behavior associate with the positive magnetoresistance observed for the thin film, we conclude that the main mechanism responsible for the MIT induced in SVO ultrathin films is electron-electron interactions other than weak localization.

For lower magnetic field and low temperature (see fig. 4a), an additional effect with a quick rise in MR is observed (which is not observed for films thicker than $7 \mathrm{~nm}$ ). This behavior can be understood taking into account the weak antilocalization (WAL) effect. WAL is observed in systems with strong spin-orbit coupling, where WAL originates from the destructive interference of coherently backscattered conduction electrons due to spin rotations. This effect has been observed in topological insulators [30], LAO/STO [31] and GaAs/GaAlAs heterostructures. The magnetoconductance $(\sigma(H, T)-\sigma(H=0, T))$ has been calculated and plotted in units of $e^{2} / \pi h$ for 
the $3 \mathrm{~nm}$ and $1.5 \mathrm{~nm}$ sample as shown in fig. $4(\mathrm{~b})$ and $5(\mathrm{~b})$, respectively. These magnetoconductance curves have been fitted using Hikami, Larkin and Nagaoka [32] equation in 2D, where in the limit of strong spin-orbit coupling $\mathrm{H}_{\mathrm{so}}>>\mathrm{H}_{\text {in }}$ :

$(\sigma(H, T)-\sigma(0, T)) /\left(\frac{e^{2}}{\pi h}\right)=\alpha\left[\ln \left(\frac{H_{i n}}{H}\right)-\psi\left(\frac{1}{2}+\frac{H_{i n}}{H}\right)\right]+A H^{2}$

Here $\psi$ is the digamma function and $H_{\text {in }}=h /\left(8 \pi e l_{\text {in }}{ }^{2}\right)$ with $l_{\text {in }}$ the inelastic mean free path.

In the equation (4), we used a $\mathrm{AH}^{2}$ parameter to take into account the effect of Lorentz force at strong magnetic fields. The fitting parameter $\alpha$ is an indication of the dominant effect between $\mathrm{WL}$, for $\alpha=-1$, and Weak Anti-Localization (WAL), for $0 \leq \alpha \leq 1 / 2$. The data were successfully fitted and the values for $\alpha$ are 0.001 for $3 \mathrm{~nm}$ and 0.004 for $1.5 \mathrm{~nm}$ corresponding to WAL effect at low magnetic fields. Interestingly, for the film of $1.5 \mathrm{~nm}$ (see fig $5 \mathrm{a}$ ), at temperatures $2 \mathrm{~K}$ and $3 \mathrm{~K}$, a competition of negative and positive magnetoresistance is observed showing contribution of weak localization when the spin-orbit coupling is of the same order than the inelastic mean free path Hso $\approx$ Hin [31]. The temperature dependences of the inelastic scattering lengths $I_{\text {in }}$ for the $3 \mathrm{~nm}$ and $1.5 \mathrm{~nm}$ sample have been extracted from the eq. 4 and are shown in fig. 4 (c) and 5 (c) respectively. $\mathrm{T}^{-\mathrm{p} / 2}$ with $\mathrm{p}=1$ variation is obtained for the $3 \mathrm{~nm}$ film which corresponds to an electron-electron collision mechanism ( $p=3$ dependency is expected for electron-phonon collision) [33]. A small deviation from $\mathrm{T}^{-1 / 2}$ is observed for the $1.5 \mathrm{~nm}$ SVO film which can be attributed to the presence of negative magnetoresistance. Nevertheless, for both these samples, $I_{\text {in }}$ is greater than the film thickness in the whole temperature range, hence we can conclude on a 2D regime at least for the inelastic mean free path even at thickness of $3 \mathrm{~nm}$.

In summary, a minimum of resistivity in the SVO transport properties appear for thicknesses below 7 $\mathrm{nm}$ and used of QCC models has evidenced that mainly REEI contribution is responsible for the upturn of the resistivity. This mechanism is due to a decrease of the state density at Fermi Energy level which leads to the formation of a gap and localization of charge carriers. In these thicknesses, the MIT is driven by e-e interactions other than localization and the reduction of the bandwidth 
strength $\mathrm{W}$. The decrease of $\mathrm{W}$ can be explained by the decrease of the effective coordination number with the thickness due to the lack of neighboring $\mathrm{V}$ ions at the interface and the surface. These results are confirming the study of SVO by Photo-Emission Spectroscopy (PES) [5] which evidenced the critical evolution of electronic state for V3d with a pseudo-gap formation for ultrathin films. Furthermore, a secondary parameter should also be taken into account because the limit for the samples with pure metallic behavior is very close to the thickness at which a strong relaxation occurs, as highlighted by RHEED analysis and discussed above in this paper (see fig $1 \mathrm{~b}$ ). With the appearance of the MIT below this thickness, strain has influence on material properties, i.e. the electronic properties of SVO similar to the effects demonstrated for $\mathrm{LaNiO}_{3}$ thin films [34] and will be discussed in the next paragraph.

Discussion toward a coherent model for MIT in SVO thin films:

In this work, we have demonstrated that MIT is controlled by confinement of the system. Furthermore, structural considerations are also important in these ultrathin films, where we observed a strong relaxation of the film at very low thickness which changes significantly the physical properties.

In our case, the electronic bandwidth $\mathrm{W}$ is controlled by the tensile strain and modifies the O-V-O bond angle as well as the $\mathrm{V}-\mathrm{O}$ stretching. By comparing structural measurements and transport properties, we observe an important correlation. As the film is fully relaxed above $50 \mathrm{~nm}$, the resistivity is metallic. Below $50 \mathrm{~nm}$, tensile strain applied on the film is increasing with the thickness reduction and induces a tetragonal distortion of the film related to an increase of the resistivity. Finally, when the films are highly strained by the substrates below $7 \mathrm{~nm}$, minimum of resistivity appears in the film. From the structural point of view, different phenomena can take place due to the influence of the stress on the perovskite: for example, tilt of the octahedra, octahedral deformation, or orbital reconstruction can be observed [35,36]. Furthermore, 2D crossover with thickness is 
related to the reduction of the effective coordination number at the interface and the surface. Indeed, in our system two interfaces break the translation symmetry through the film thickness and change the chemical bond covalence. As calculated by Density Functional Theory (DFT) [36], the interface STO-SVO tends to induce an orbital reconstruction. The hybridizations of the $d_{x z}$ and $d_{y z}$ orbitals of $\mathrm{Ti}$ and $\mathrm{V}$ along the $\mathrm{z}$ axis are of lower energy and should be formed. On the contrary, at the interface of $V$ with the surface, hopping in vertical direction is reduced and $d_{x y}$ is reinforced. Moreover, confinement effect for films below $7 \mathrm{~nm}$ leads to new properties associated with the apparition of spin-orbit coupling. This is in perfect agreement with the studies by Local-Density Approximation (LDA)+U approach [7] which have shown that tetragonal strain and spin-orbit coupling controlled the strong interaction effects with orbital ordering. Indeed, novel and complex behaviors can be observed at heterointerfaces and low dimensional systems and detailed structural analysis with synchrotron or ultraSTEM may contribute to understand the interplay between structure and electronic properties and the role of the strain on the film properties in order to control or induce new properties of low dimensional SVO based systems and superlattices at the vicinity of Mott type-MIT [37-39].

\section{Summary}

In summary, transport properties in ultrathin SVO films present a thickness-induced MIT with a crossover from 3D to 2D calculated from QCC and VRH models. Correlations with in-plane and out-ofplane structural stress evolutions show the existence of a $7 \mathrm{~nm}$ relaxation thickness corresponding to the metallic state limit. A detailed investigation of conductivity mechanisms permits to demonstrate that REEI mechanism is responsible for the upturn of the resistivity. WAL effect also appears for low SVO thickness due to strong spin-orbit coupling and a mechanism of electron-electron collisions in 2D system is shown from the temperature dependence of inelastic scattering length. 


\section{Aknowledgments:}

This work was supported by lle de France region for magneto-transport measurements ("NOVATECS"

C'Nano IdF project n`IF-08-1453/R). Preliminary experimental support by W. Prellier, U. Luders, A. David and $\mathrm{H}$. Rotella is acknowledged. 


\section{References}

[1] M. Kestigian, J. G. Dickinson, and R. Ward, J. Am. Chem. Soc. 79, 5598 (1957).

[2] M. J. Rey, P. Dehaudt, J. C. Joubert, B. Lambert-Andron, M. Cyrot, and F. Cyrot-Lackmann, J. Solid State Chem. 86, 101 (1990).

[3] P. Dougier, J. C. C. Fan, and J. B. Goodenough, J. Solid State Chem. 14, 247 (1975).

[4] T. Maekawa, K. Kurosaki, and S. Yamanaka, J. Alloys Compd. 426, 46 (2006).

[5] K. Yoshimatsu, T. Okabe, H. Kumigashira, S. Okamoto, S. Aizaki, A. Fujimori, and M. Oshima, Phys. Rev. Lett. 104, 147601 (2010).

[6] Y. C. Lan, X. L. Chen, and M. He, J. Alloys Compd. 354, 95 (2003).

[7] V. Pardo and W. E. Pickett, Phys. Rev. B 81, 245117 (2010).

[8] J. Hubbard, Proc. R. Soc. Lond. Math. Phys. Eng. Sci. 276, 238 (1963).

[9] I. H. Inoue, O. Goto, H. Makino, N. E. Hussey, and M. Ishikawa, Phys. Rev. B 58, 4372 (1998).

[10] H. Makino, I. H. Inoue, M. J. Rozenberg, I. Hase, Y. Aiura, and S. Onari, Phys. Rev. B 58, 4384 (1998).

[11] K. Yoshimatsu, K. Horiba, H. Kumigashira, T. Yoshida, A. Fujimori, and M. Oshima, Science 333, 319 (2011).

[12] G. Herranz, F. Sánchez, J. Fontcuberta, V. Laukhin, J. Galibert, M. V. García-Cuenca, C. Ferrater, and M. Varela, Phys. Rev. B 72, 14457 (2005).

[13] R. Scherwitzl, S. Gariglio, M. Gabay, P. Zubko, M. Gibert, and J.-M. Triscone, Phys. Rev. Lett. 106, 246403 (2011).

[14] J. Xia, W. Siemons, G. Koster, M. R. Beasley, and A. Kapitulnik, Phys. Rev. B 79, 140407 (2009).

[15] A. Matthiessen and C. Vogt, Philos. Trans. R. Soc. Lond. 154, 167 (1864).

[16] P. A. Lee and T. V. Ramakrishnan, Rev. Mod. Phys. 57, 287 (1985).

[17] G. M. Minkov, A. V. Germanenko, O. E. Rut, A. A. Sherstobitov, and B. N. Zvonkov, Phys. Rev. B 79, 235335 (2009).

[18] D. Pesquera, X. Marti, V. Holy, R. Bachelet, G. Herranz, and J. Fontcuberta, Appl. Phys. Lett. 99, 221901 (2011).

[19] R. t Shannon, Acta Crystallogr. A 32, 751 (1976).

[20] N. Junqua and J. Grilhé, Thin Solid Films 250, 37 (1994).

[21] E. Popova, B. Warot-Fonrose, F. Bonell, S. Andrieu, Y. Dumont, B. Berini, A. Fouchet, and N. Keller, Surf. Sci. 605, 1043 (2011).

[22] I. H. Inoue, H. Makino, I. Hase, M. Ishikawa, N. E. Hussey, and M. J. Rozenberg, Phys. B Condens. Matter 237, 61 (1997).

[23] V. Giannakopoulou, P. Odier, J. M. Bassat, and J. P. Loup, Solid State Commun. 93, 579 (1995).

[24] W. Noun, B. Berini, Y. Dumont, P. R. Dahoo, and N. Keller, J. Appl. Phys. 102, 63709 (2007).

[25] B. Berini, N. Keller, Y. Dumont, E. Popova, W. Noun, M. Guyot, J. Vigneron, A. Etcheberry, N. Franco, and R. M. C. da Silva, Phys. Rev. B 76, 205417 (2007).

[26] M. Gu, S. A. Wolf, and J. Lu, Adv. Mater. Interfaces 1, 1300126 (2014).

[27] J. A. Moyer, C. Eaton, and R. Engel-Herbert, Adv. Mater. 25, 3578 (2013).

[28] M. P. Warusawithana, C. Richter, J. A. Mundy, P. Roy, J. Ludwig, S. Paetel, T. Heeg, A. A. Pawlicki, L. F. Kourkoutis, M. Zheng, M. Lee, B. Mulcahy, W. Zander, Y. Zhu, J. Schubert, J. N. Eckstein, D. A. Muller, C. S. Hellberg, J. Mannhart, and D. G. Schlom, Nat. Commun. 4, 2351 (2013).

[29] W. Brenig, G. H. Döhler, and H. Heyszenau, Philos. Mag. 27, 1093 (1973).

[30] M. Liu, J. Zhang, C.-Z. Chang, Z. Zhang, X. Feng, K. Li, K. He, L. Wang, X. Chen, X. Dai, Z. Fang, Q.K. Xue, X. Ma, and Y. Wang, Phys. Rev. Lett. 108, 36805 (2012).

[31] A. Caviglia, M. Gabay, S. Gariglio, N. Reyren, C. Cancellieri, and J.-M. Triscone, Phys Rev Lett 104, 126803 (2010).

[32] S. Hikami, A. I. Larkin, and Y. Nagaoka, Prog. Theor. Phys. 63, 707 (1980).

[33] G. Bergmann, Phys. Rep. 107, 1 (1984). 
[34] J. Son, P. Moetakef, J. M. LeBeau, D. Ouellette, L. Balents, S. J. Allen, and S. Stemmer, Appl. Phys. Lett. 96, 62114 (2010).

[35] J. M. Rondinelli and N. A. Spaldin, Adv. Mater. 23, 3363 (2011).

[36] Z. Zhong, P. Wissgott, K. Held, and G. Sangiovanni, EPL Europhys. Lett. 99, 37011 (2012).

[37] U. Lüders, W. C. Sheets, A. David, W. Prellier, and R. Frésard, Phys. Rev. B 80, 241102 (2009).

[38] W. C. Sheets, P. Boullay, U. Lüders, B. Mercey, and W. Prellier, Thin Solid Films 517, 5130 (2009).

[39] D. H. Kim, D.-W. Kim, B. S. Kang, T. W. Noh, D. R. Lee, K.-B. Lee, and S. J. Lee, Solid State Commun. 114, 473 (2000). 
Figures

FIG. 1. (Color online) (a) Dependence of out-of-plane lattice parameter, calculated from XRD measurements, on film thickness. STO red and SVO green lines correspond to bulk values of the lattice constant of STO and SVO, respectively. The dashed black line corresponds to $\mathrm{SrVO}_{2.90}$ lattice parameter ${ }^{3}$. The inset presents (200) XRD pattern (black) and fit using a dynamical model (dashed navy). (b) (110) Interplane distance evolution with film thickness. Red and green lines correspond to theoretical values in STO and SVO, respectively. (inset) (110) RHEED patterns realized in-situ for different thin film growth time at $0 \mathrm{sec}$ (STO) and $30 \mathrm{sec}$ (4 nm SVO film).

FIG. 2. (Color online) (a) Resistivity versus temperature for different film thicknesses. The minima are indicated by arrows and corresponding temperatures. (b) Resistivity evolution as a function of temperature for a metallic $115 \mathrm{~nm}$ thick SVO film. (c) Variation of the carrier density and mobility from Hall measurements for 115 and $3 \mathrm{~nm}$ thick film versus temperature. (d) Resistivity versus temperature for a $7 \mathrm{~nm}$ film and 3D model calculated (blue) for the low-temperature behavior. The inset shows the sheet conductance as a function of $\operatorname{lnT}$ for the same film and $2 \mathrm{D}$ models for $\mathrm{p}=1$ (red line) and $p=3$ (blue dashed line).

FIG. 3. (Color online) (a) Resistivity versus temperature for a $3 \mathrm{~nm}$ film and 3D model calculated (blue) for the low-temperature behavior. In inset sheet conductance is presented as a function of InT for the same film and 2D models for $p=1$ (red line) and $p=3$ (blue dashed line). (b) Logarithm of sheet conductance as a function of $1 / \mathrm{T}^{1 / 3}$ for a $1.5-2 \mathrm{~nm}$ SVO film. The red line is the linear fit corresponding to 2D VRH model. The inset shows the resistivity versus temperature for the same sample.

FIG. 4. (Color online) (a) Magnetoresistance (MR) in perpendicular field for a $3 \mathrm{~nm}$ SVO film at different temperatures and fits with $\mathrm{H}^{2}$ dependence in magenta. (b) Magnetoconductance versus magnetic field and fits in red lines calculated with Hikami, Larkin and Nagaoka equation. (c) 
Temperature dependence of the inelastic scattering length extracted from magnetoconductance fits. Red dashed line and green line correspond to $\mathrm{T}^{-3 / 2}$ and $\mathrm{T}^{-1 / 2}$ variation, respectively.

FIG. 5. (Color online) (a) Magnetoresistance in perpendicular field for a $1.5 \mathrm{~nm}$ SVO film at different temperatures. (b) Magnetoconductance versus magnetic field and fits in red lines calculated with Hikami, Larkin and Nagaoka equation. (c) Temperature dependence of the inelastic scattering length extracted from magnetoconductance fits. Red dashed line and green line correspond to $T^{-3 / 2}$ and $T^{-1 / 2}$ variation, respectively.

Table $1 . \mathrm{SrVO}_{3}$ thin films parameters $\rho_{0}, A$ for a quadratic Fermi liquid model of the resistivity $\left(\rho=\rho_{0}+A * T^{2}\right)$ 

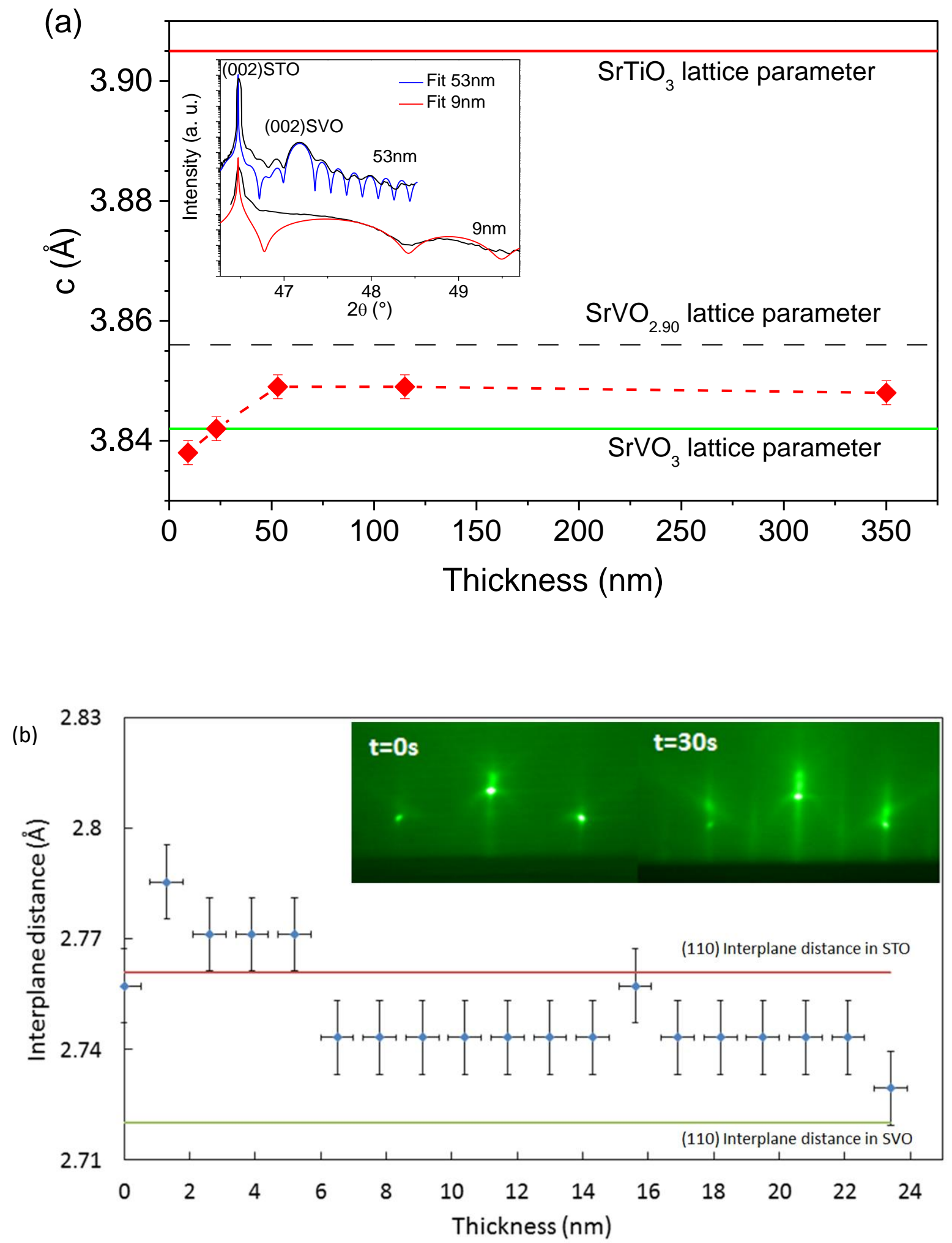

FIG. 1. (a) and (b) 
Fig 2

(a)

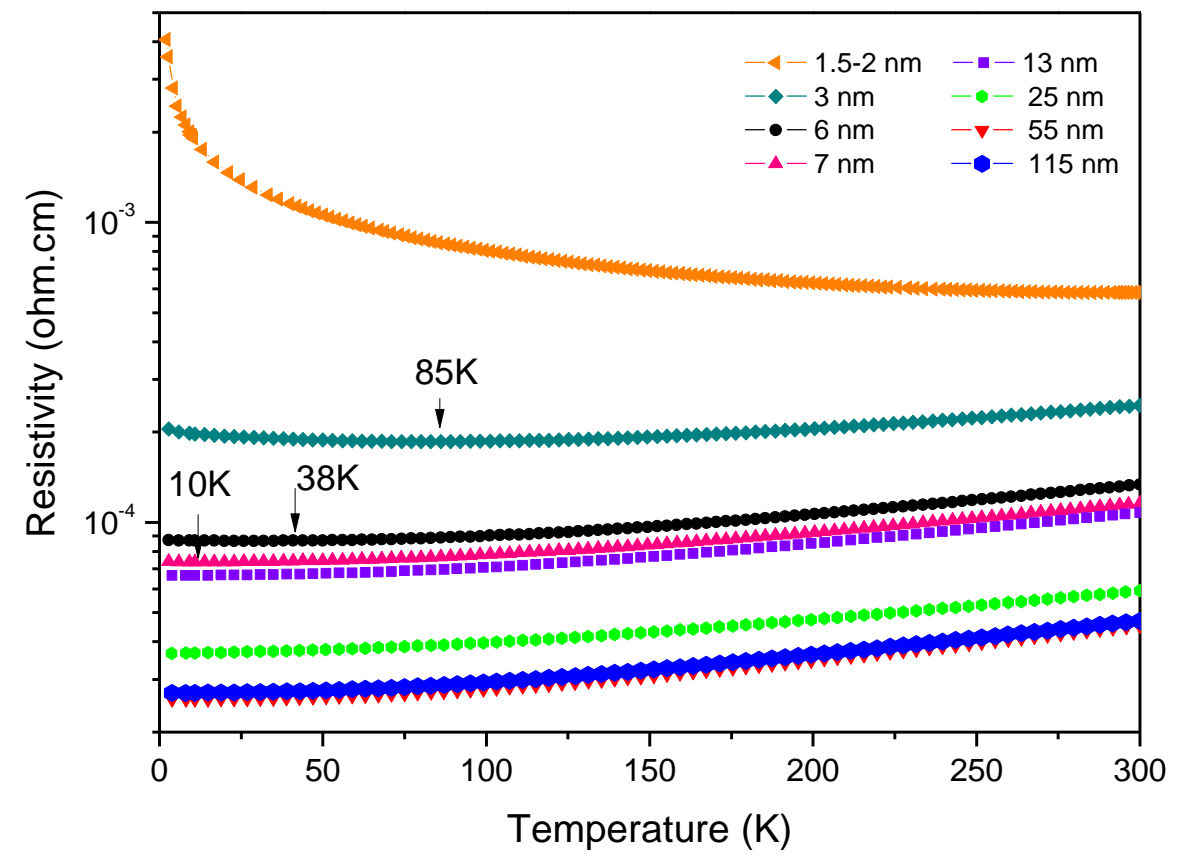

(b)

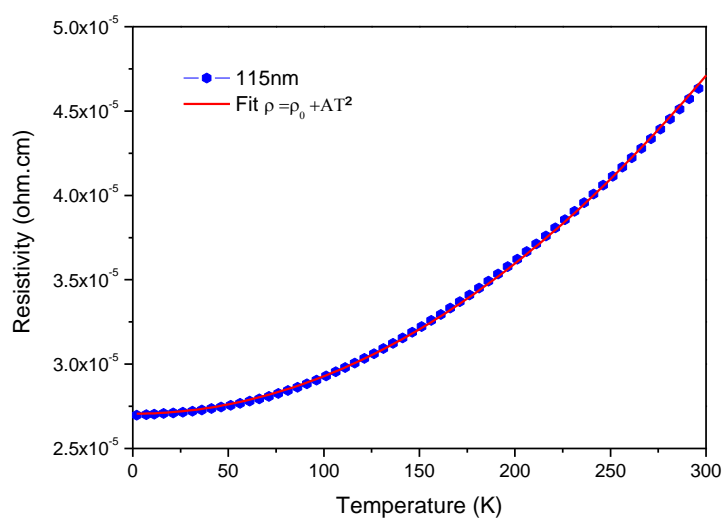

(c)

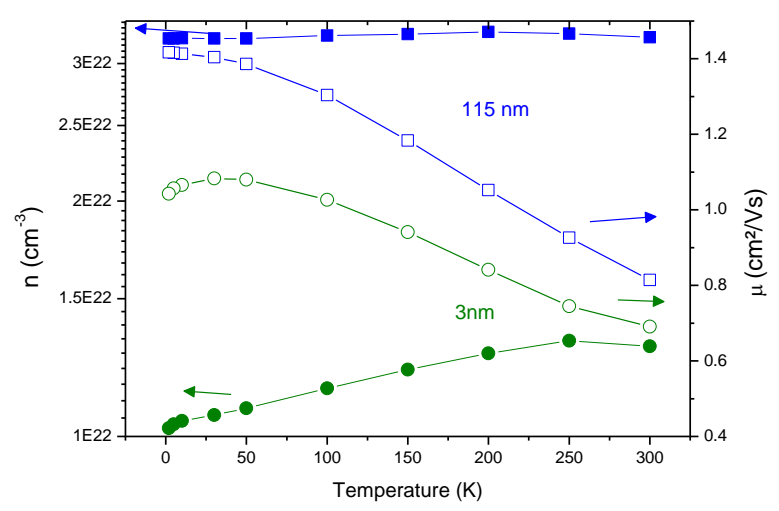




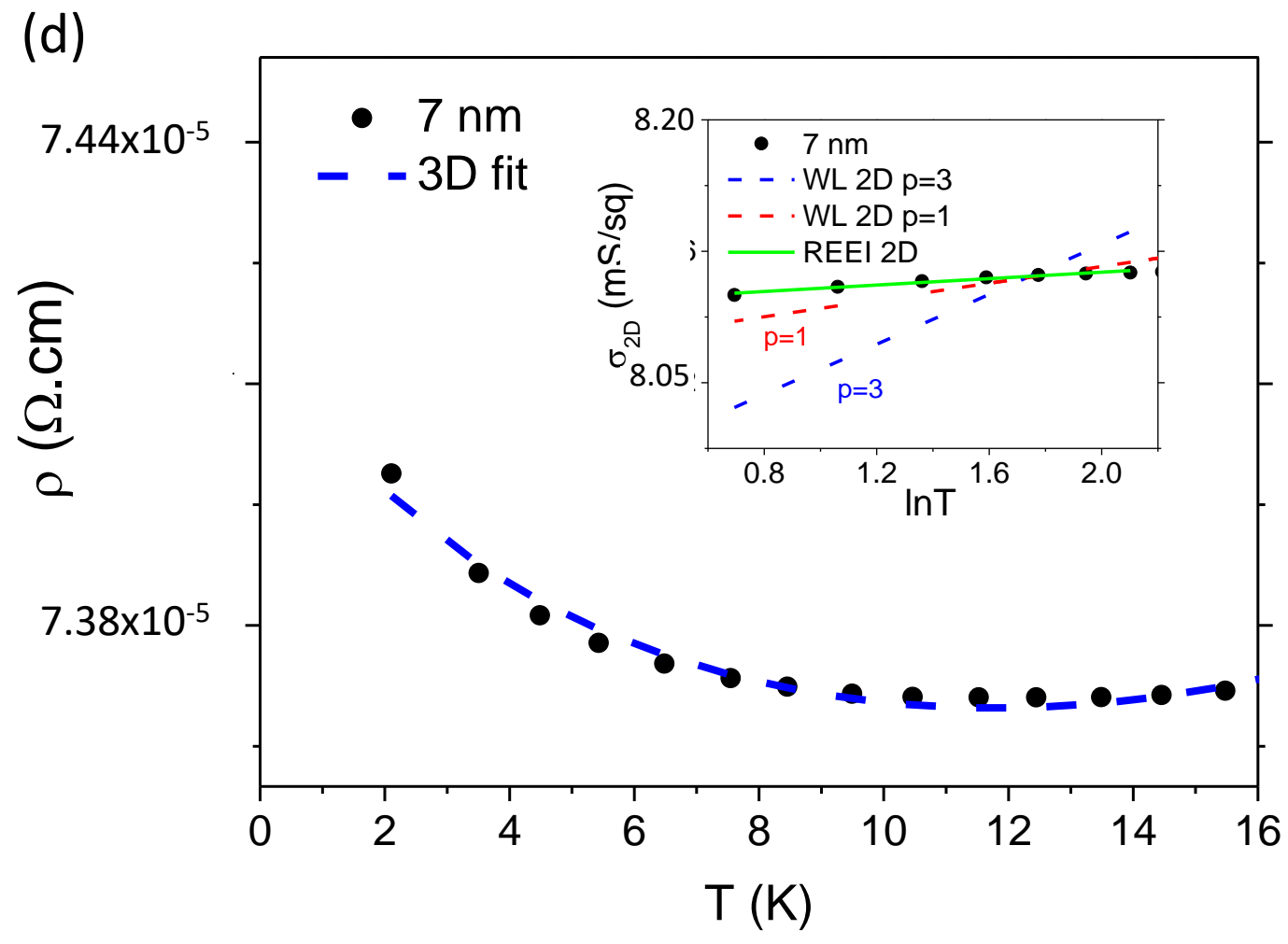

FIG. 2. (a), (b), (c ) and (d). 
Fig 3

(a)

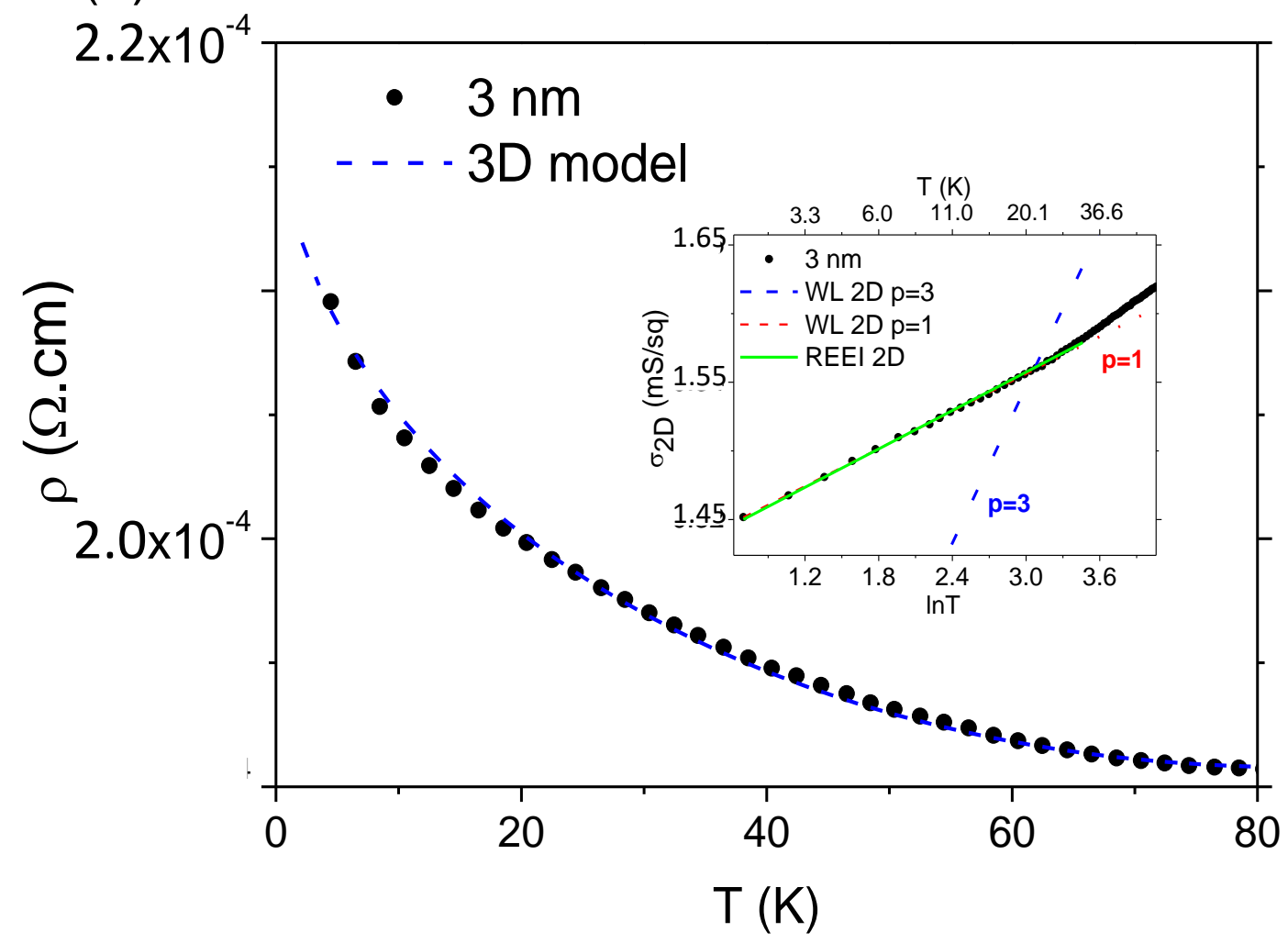


(b)

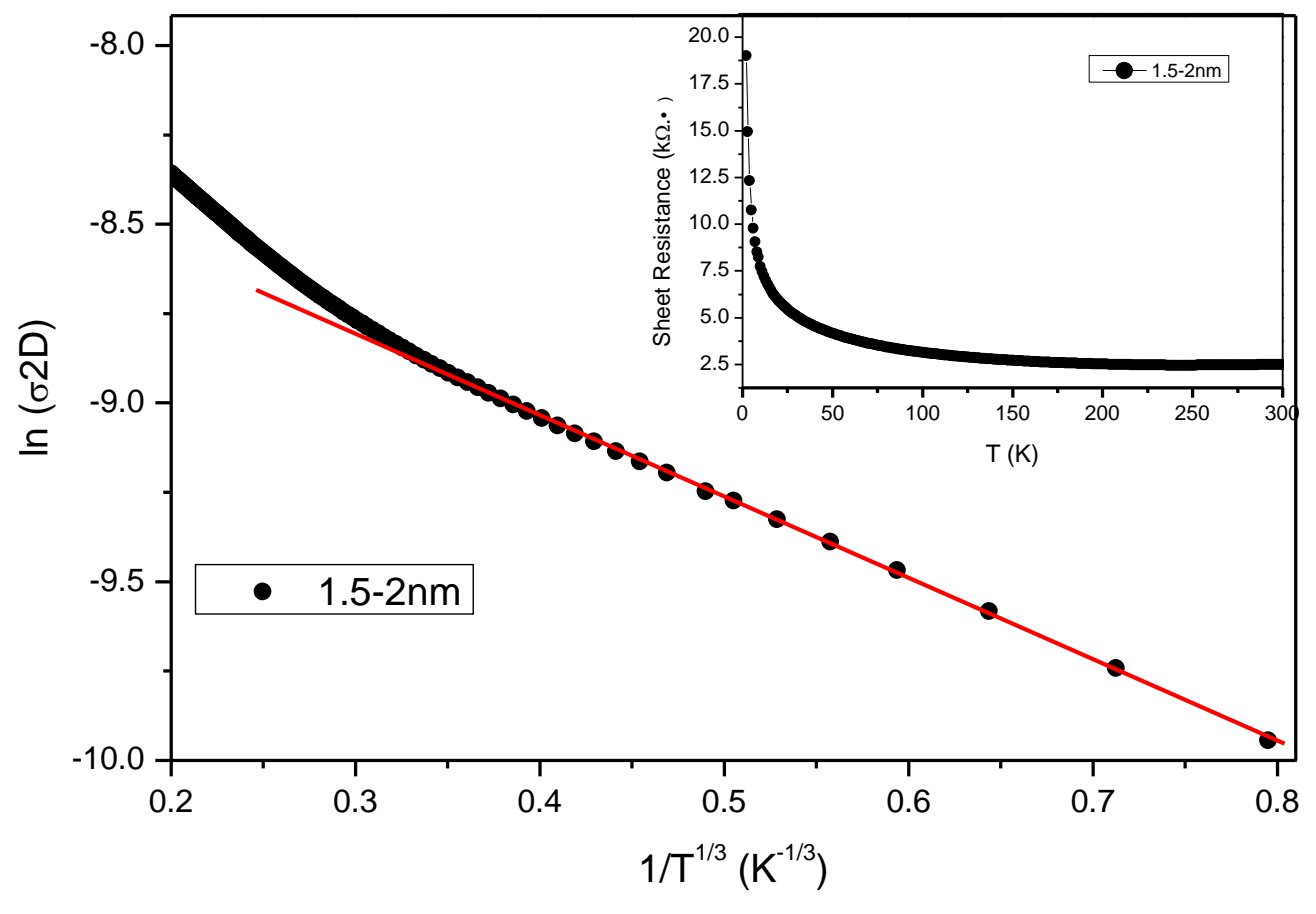

FIG. 3. (a) and (b) 
Fig 4
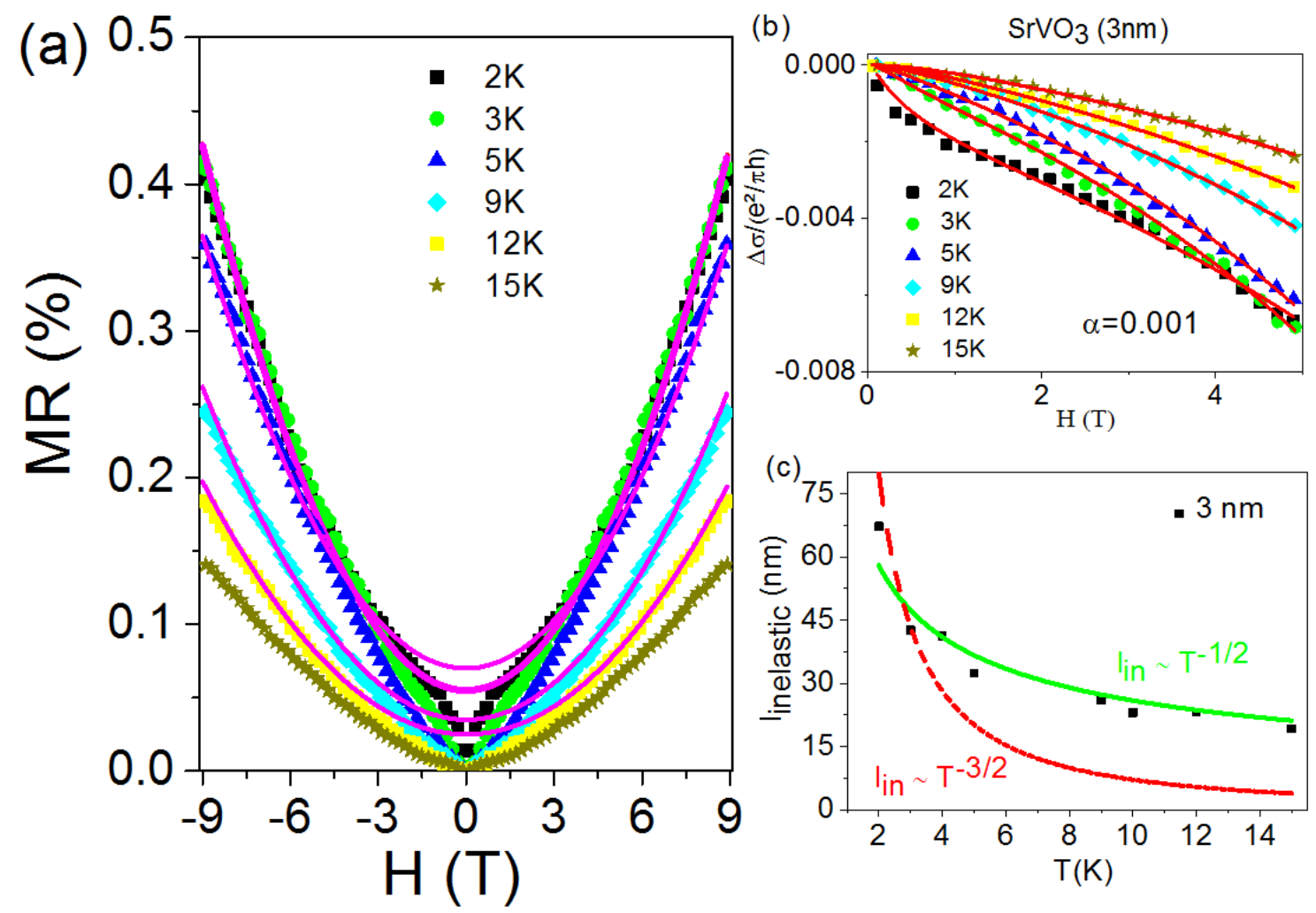

FIG. 4.

FIG. 5.
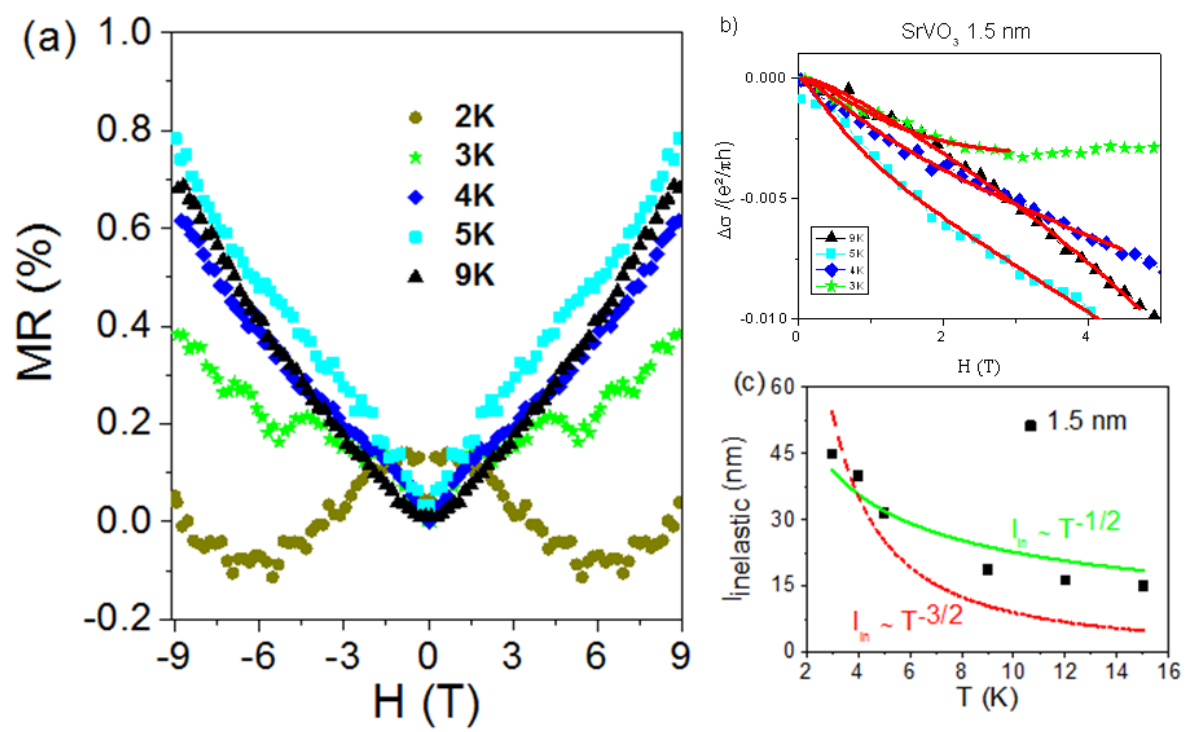

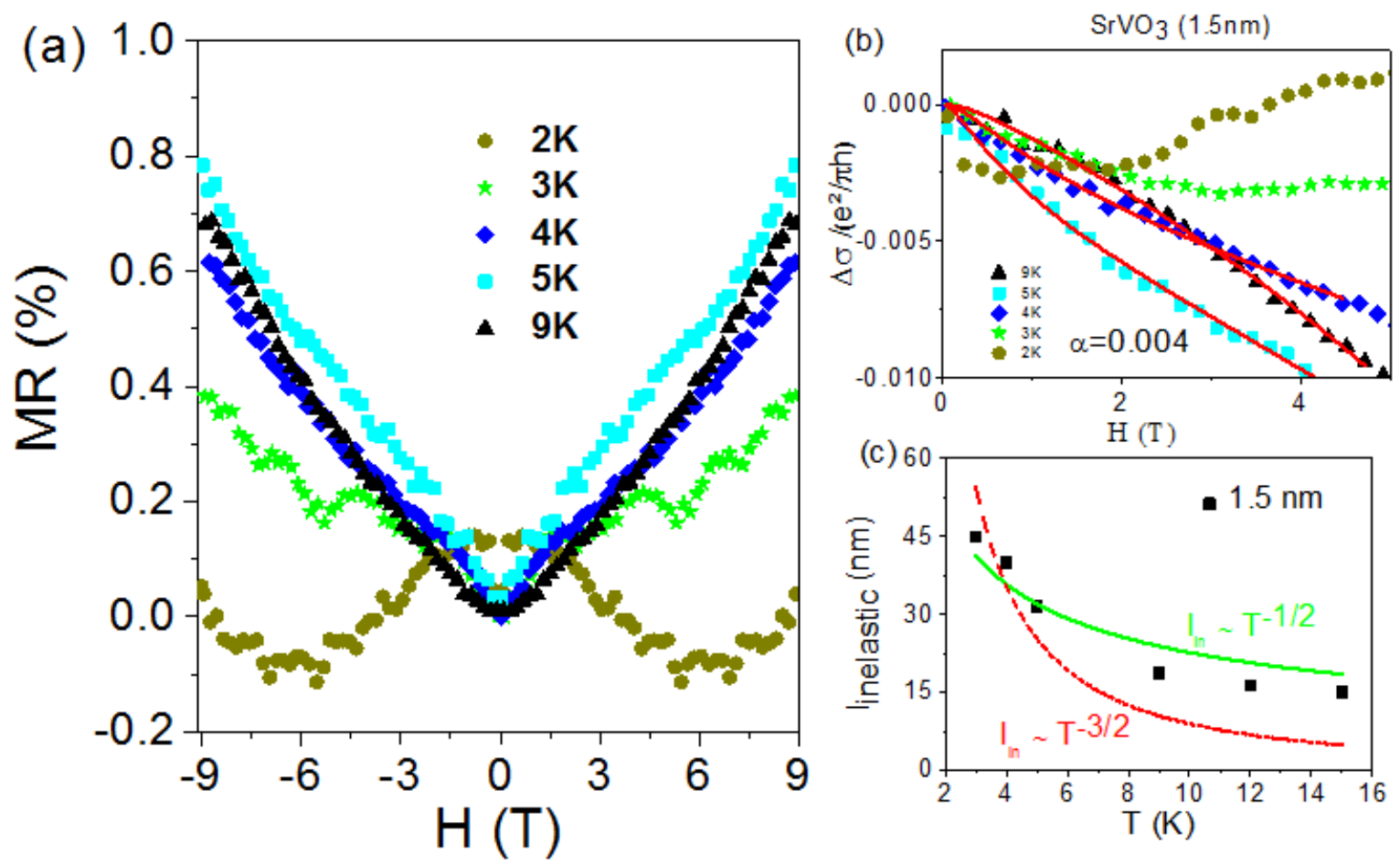

FIG. 5.

Table 1.

\begin{tabular}{|c|c|c|}
\hline Film thickness (nm) & $\boldsymbol{\rho}_{\mathbf{0}}(\mathbf{\Omega . c m})$ & $\mathbf{A}\left(\mathbf{\Omega . c m} \cdot \mathbf{K}^{-2}\right)$ \\
\hline $\mathbf{1 1 5}$ & $2.7^{*} 10^{-5}+/-1^{*} 10^{-6}$ & $2.2^{*} 10^{-10}+/-1^{*} 10^{-11}$ \\
\hline $\mathbf{5 5}$ & $2.7^{*} 10^{-5}+/-1^{*} 10^{-6}$ & $2.2^{*} 10^{-10}+/-1^{*} 10^{-11}$ \\
\hline $\mathbf{2 5}$ & $3.7^{*} 10^{-5}+/-1^{*} 10^{-6}$ & $2.5^{*} 10^{-10}+/-1^{*} 10^{-11}$ \\
\hline $\mathbf{1 3}$ & $6.6^{*} 10^{-5}+/-1^{*} 10^{-6}$ & $4.6 * 10^{-10}+/-1^{*} 10^{-11}$ \\
\hline $\mathbf{7}$ & $7.3^{*} 10^{-5}+/-1^{*} 10^{-6}$ & $4.7^{*} 10^{-10}+/-1^{*} 10^{-11}$ \\
\hline $\mathbf{3}$ & $17.4^{*} 10^{-5}+/-1^{*} 10^{-6}$ & $7.8^{*} 10^{-10}+/-1^{*} 10^{-11}$ \\
\hline
\end{tabular}



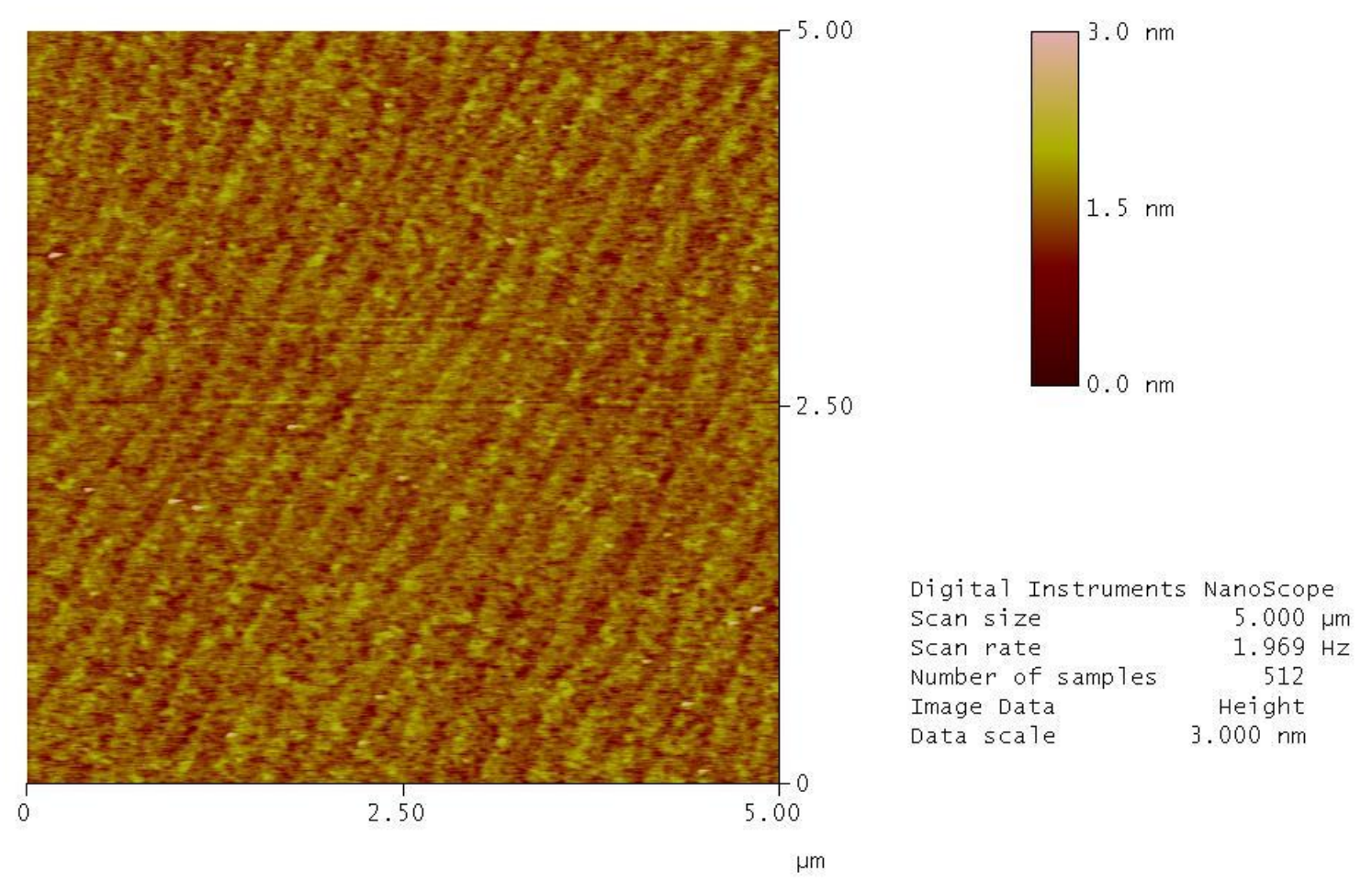

FIG. SI-1: AFM topographic images $(5 \mu \mathrm{m} \times 5 \mu \mathrm{m})$ of the $3 \mathrm{~nm}$ SVO thin film.

The surface morphology has been studied with AFM. The AFM topographic image is shown on the figure SI-1 for the SVO film of $3 \mathrm{~nm}$. Terrasses can be observed corresponding to the same morphology than the STO substrate. The rough mean square of the film is $0.2 \mathrm{~nm}$. 